\title{
Telomere behavior in a hybrid yeast
}

\author{
Cell Research (2009) 19:910-912. doi: 10.1038/cr.2009.65; published online 9 June 2009
}

\section{Dear Editor,}

Telomeres and the protein/RNA complexes involved in maintaining them are rapidly evolving systems across eukaryotes. Using two Saccharomyces species, among $S$. cerevisiae and $S$. bayanus, we provide evidence that the telomere systems of these two closely related yeasts have evolved significantly apart and that the gene in one species cannot maintain the set-point of telomere length of the other species in the hybrid.

The sequence of telomere repeats, abbreviated $\mathrm{C}_{1-3} \mathrm{~A} /$ $\mathrm{TG}_{1-3}$, of the related Saccharomyces sensu stricto species are completely conserved. However, the lengths of the telomere repeats vary greatly even among different strains within the same species $[1,2]$. The sequences adjacent to the repeats are also rapidly evolving both within and among Saccharomyces species [3]. For example, the subtelomeric repeat, called the $\mathrm{Y}^{\prime}$ element, is present in S. cerevisiae, but absent from S. bayanus [4]. Although the $\mathrm{X}$ element, another subtelomeric repeat, is present in both species, the primary sequences have diverged both within and between the two species [4]. Recent whole genome sequencing of Saccharomyces sensu stricto species, including S. paradoxus, S. mikatae, and S. bayanus, has revealed that in addition to the rapid evolution of $\mathrm{X}$ and $\mathrm{Y}^{\prime}$ elements, the gene families found within the subtelomeric regions also show significant differences in different species [5].

In addition to the divergence of subtelomeric regions, proteins and RNA involved in maintaining the terminal telomeric repeats also exhibit high degrees of sequence divergence. For example, the RNA components of the $S$. cerevisiae and S. bayanus telomerases share only $\sim 43 \%$ nucleic acid identity. The divergence of the telomere system in closely related yeast species suggests that proteins and/or RNA involved in regulating telomere length in one species may not be able to maintain the set-point of the telomere length found in other species.

To test this possibility, we studied the telomere behavior in a hybrid yeast between $S$. cerevisiae and $S$. bayanus. We first created heterothallic $S$. bayanus strains by disrupting the HO locus of a wild homothallic diploid $S$. bayanus derived from the sequenced strain. Haploids of opposite mating types from diploid $S$. bayanus and $S$. cerevisiae were mated to create reciprocal hybrid yeasts (BC11 and BC12) (Supplementary information, Data $\mathrm{S} 1)$. The diploid S. bayanus grew well at low temperatures but could not grow at $37^{\circ} \mathrm{C}$. The hybrid yeasts, on the other hand, exhibited vigorous growth at all temperatures tested (Figure 1A).

To determine the telomere length, genomic DNAs from the hybrids as well as their corresponding parental diploids were digested with XhoI, separated by gel electrophoresis, and probed with a telomere-specific probe (Figure 1B). The S. cerevisiae diploid (CC5) exhibited a typical pattern of DNA fragments derived from hybridization to many telomeric fragments and to several subtelomeric repeats. The shortest fragments (arrow in Figure 1B) represent the $\mathrm{Y}^{\prime}$-containing telomeres. In contrast, all of the telomeres in the $S$. bayanus diploid (BB9) remained in large DNA fragments following XhoI digestion. This allowed us to unequivocally identify a number of $S$. cerevisiae telomeres. These $S$. cerevisiae telomeres, especially the $\mathrm{Y}^{\prime}$ telomeres, appeared shorter in the hybrids than those in the parental $S$. cerevisiae diploids (Figure 1B). Interestingly, although shorter, these telomeres were stable in the hybrids after $\sim 500$ doublings (data not shown), suggesting that they were maintained in this short length equilibrium.

The clearly identifiable $S$. cerevisiae telomeres allowed us to test whether factors that regulate telomere length maintenance in either $S$. cerevisiae or $S$. bayanus have evolved significantly apart so that they have become less efficient in maintaining the set-point of the length of telomeres of the other species. Since the Y'-containing telomeres are most reliable in determining telomeric length differences among $S$. cerevisiae strains, we focused our attention to these telomeres. TLC1, the RNA component of the yeast telomerase, and EST1, a protein involved in recruiting the telomerase to telomeres, both exhibit a high degree of sequence divergence between the two species, sharing $\sim 43 \%$ and $60 \%$ identity at the nucleotide and amino acid level, respectively. Therefore, we asked whether deleting species-specific alleles of these genes in the hybrid would have the same or different effects on the length of the $S$. cerevisiae $\mathrm{Y}^{\prime}$ telomeres. We first de- 
A
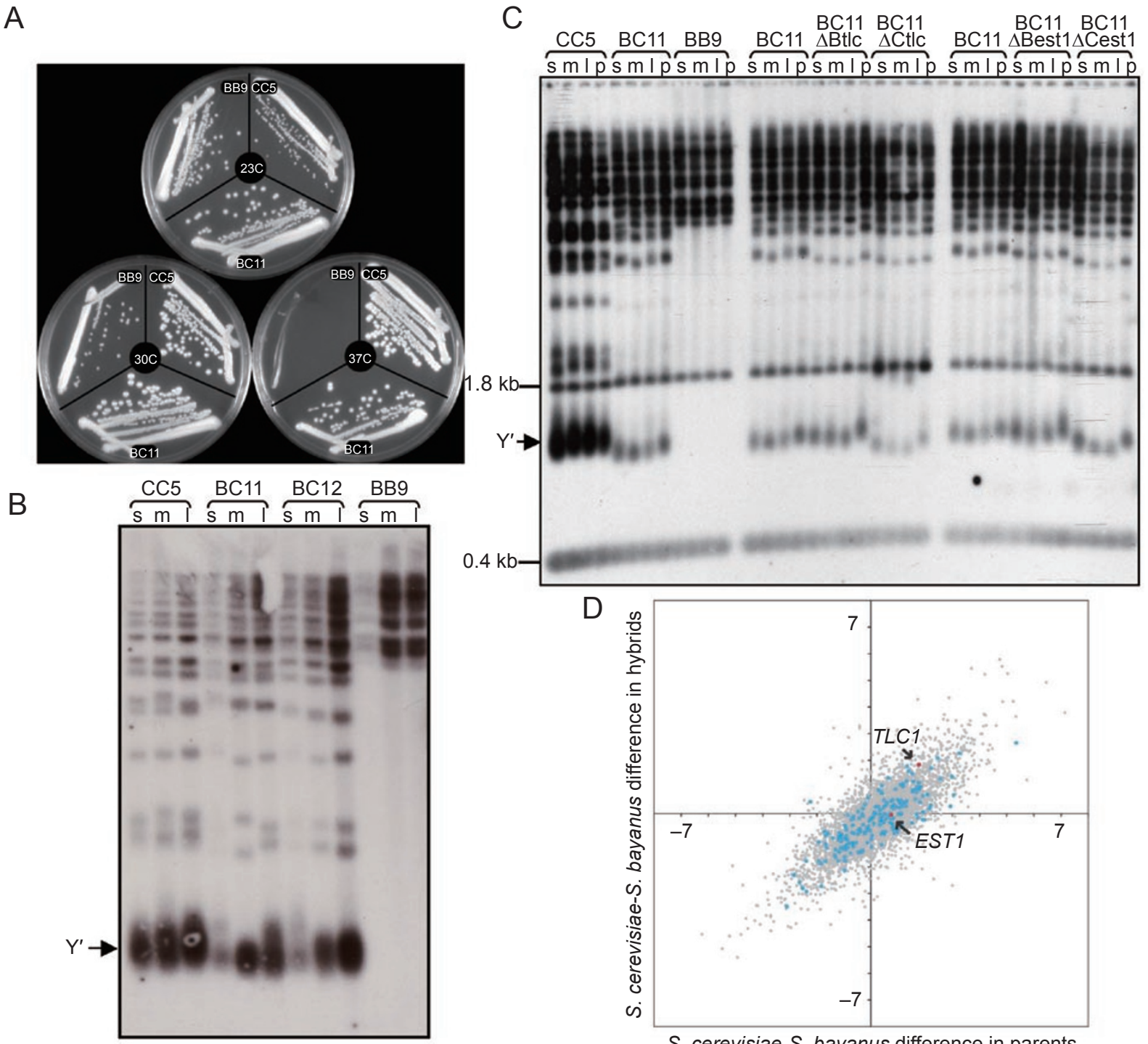

D

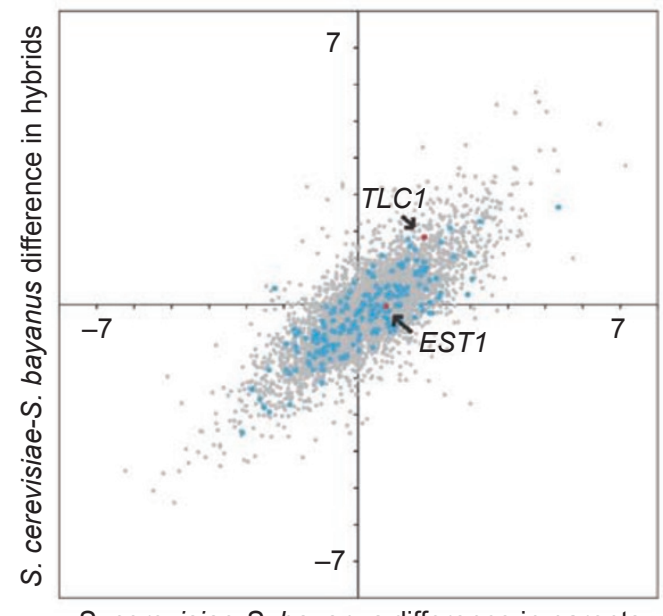

S. cerevisiae-S. bayanus difference in parents

Figure 1 (A) Hybrid BC11 yeasts grew well over a wider temperature range than the diploid S. bayanus strain. (B) Genomic DNA isolated from small (s), medium (m), and large (I) colonies of $S$. cerevisiae diploid (CC5), S. bayanus diploid (BB9), and the reciprocal hybrids (BC11 and $\mathrm{BC} 12)$ between these two diploids were digested by Xhol and analyzed by Southern blotting. Arrow indicates the $S$. cerevisiae $Y^{\prime}$ telomeres. (C) A preference for $S$. cerevisiae TLC1 and EST1 in maintaining the length of $Y^{\prime}$ telomeres in the hybrid. Genomic DNA from individual small $(s)$, medium $(m)$, or large (I) colonies, or populations $(p)$ of control strains (CC5, BC11, and BB9) or deletion strains, BC11 $\triangle B$ Best1 (deleting S. bayanus EST1), BC11 $\triangle \mathrm{Btl}$ (deleting $S$. bayanus TLC1), BC11 $\triangle$ Cest1 (deleting S. cerevisiae EST1), and BC11 $\triangle$ Ctlc (deleting S. cerevisiae TLC1), were digested using Xhol and analyzed by Southern blotting using the telomere probe. The 1.8 and $0.4 \mathrm{~kb}$ fragments containing the telomere repeats were generated by digesting the telomere repeat-containing plasmid pYTLA.1 and mixing with the genomic DNA as size markers. Arrow points to the $Y^{\prime}$ telomeres. (D) Microarray analysis of the expression of genes involved in regulating telomere length. Using a custom-designed, species-specific, spotted oligonucleotide microarray, we measured the expression level of each gene versus a matched DNA reference for each parent species and the hybrid. Each strain was grown to midlog phase at $23^{\circ} \mathrm{C}$ and $30^{\circ} \mathrm{C}$, and both experiments were averaged for further analysis. $\log _{2}$ expression levels of orthologous genes were subtracted from one another to yield expression differences between the parental diploids or differences between alleles in the hybrid. These values graphed as a scatter plot show a strong correlation, indicating that most genes are expressed at similar levels in the parent as in the hybrid. Orthologs clustered near the origin (e.g. EST1, marked in red) show similar levels between species, while those in the upper right (e.g. TLC1, marked in red) or lower left tails are expressed higher in one species than the other. Additional telomere-related genes are highlighted in blue. All other genes are shown in gray. All microarray data are available from the Princeton Microarray Database (puma.princeton.edu) and GEO (http://www. ncbi.nlm.nih.gov/projects/geo/ accession: GSE15877). 
leted the S. bayanus allele of either TLC1 or EST1 in the hybrid (Supplementary information, Data S1). Compared to the $\mathrm{Y}^{\prime}$ telomeres of the undeleted hybrids, the lengths of hybrid $S$. cerevisiae $\mathrm{Y}^{\prime}$ telomeres were unaffected by the deletions (Figure 1C). However, when the S. cerevisiae alleles of TLC1 or EST1 were deleted in the hybrid, the $S$. cerevisiae $\mathrm{Y}^{\prime}$ telomeres became shorter compared to controls (Figure 1C). Again, these short $Y^{\prime}$ telomeres were stable after $\sim 500$ doublings (data not shown), suggesting that the $S$. bayanus TLC1 and EST1 were able to maintain the $\mathrm{Y}^{\prime} S$. cerevisiae telomeres at the short length equilibrium in the hybrid.

An obvious interpretation of the above results is that the $S$. bayanus TLC1 and EST1 have diverged significantly from those of $S$. cerevisiae and consequently have become unable to maintain the set-point length of the $S$. cerevisiae telomeres. However, it is also possible that the hybrid exhibits allelic bias in gene expression. If the telomere maintenance genes are expressed preferentially by the $S$. cerevisiae alleles, deleting these alleles could have a strong effect on the length of telomeres. To test this latter possibility, we used microarray analyses to compare gene expression in the parental diploid strains, CC5 and BB9, and in the hybrid BC11 strain (Supplementary information, Data S1).

Measured in exponentially growing cells, gene expression differed somewhat between the two species, and these differences were largely retained in the hybrid (Figure 1D). In order to see if telomere-related genes were more likely to show expression differences than other genes, we generated a list of 202 genes (Supplementary information, Table S1) that had previously been associated with telomere biology or were reported to influence telomere length maintenance $[2,6]$. Though some of these genes were expressed at higher levels in one species' genome than the other (Figure 1D), the distribution mean was not statistically different from non-telomererelated genes ( $t$-test), and in fact the variance was lower for these genes than for the rest of the genome. Thus, there were no systematic differences in expression of genes affecting telomeres.

Looking at individual genes, $S$. cerevisiae $T L C 1$ was expressed at three-fold higher levels in $\mathrm{CC} 5$ than in BB9. In the hybrid, the $S$. cerevisiae $T L C 1$ allele retained its higher expression level than the S. bayanus TLC1 allele (Figure 1D). EST1, however, did not exhibit allelic gene expression bias in the parental strains or the hybrid (Figure 1D). These analyses show that at least the preference for S. cerevisiae EST1 in maintaining the length of the cerevisiae-specific $\mathrm{Y}^{\prime}$ telomeres in the hybrid is not due to allelic bias in gene expression. This suggests that telomere systems in two closely related species have diverged significantly such that the function to maintain the set-point telomere length of certain genes in one species cannot be fully substituted by the corresponding genes in another species. The divergent telomere systems could be one of the driving forces for chromosome rearrangements, gene deletion, or duplication during evolution of the hybrid yeast.

\section{Acknowledgments}

We thank Ms Margaret Hoang (Carnegie Institution of Washington) for sharing hybrid strains, Dr Ed Louis (The University of Nottingham) for generous help with reagents, and the members of the Zheng lab for helpful comments. This work was supported by NIH P50 GM071508 to the Lewis-Sigler Institute and Howard Hughes Medical Institute. YZ and DEK are investigators of HHMI.

Ona C Martin ${ }^{1}$, Christopher G DeSevo ${ }^{2}$, Benjamin Z Guo ${ }^{3}$, Douglas E Koshland ${ }^{1}$, Maitreya J Dunham ${ }^{2,4}$, Yixian Zheng ${ }^{1}$

${ }^{I}$ Department of Embryology, Carnegie Institution of Washington and Howard Hughes Medical Institute, Baltimore, MD 21218, USA; ${ }^{2}$ Lewis-Sigler Institute, Princeton University, Princeton, NJ 08544; ${ }^{3}$ Harvard College, University Hall, Cambridge, MA 02138; ${ }^{4}$ Department of Genome Sciences, University of Washington, Seattle, WA 98195-5065

Correspondence: Yixian Zheng

E-mail: Zheng@ciwemb.edu

\section{References}

1 Dandjinou AT, Levesque N, Larose S, et al. A phylogenetic based secondary structure for the yeast telomere RNA. Curr Biol 2004; 14:1148-1158.

2 Gatbonton T, Imbesi M, Nelson M, et al. Telomere length as a quantitative trait: genome-wide survey and genetic mapping of telomere length-control genes in yeast. PLoS Genet 2006; 2:e35.

3 Mondoux MA, Zakian VA. Telomere position effect: silencing near the end. In: T de Lange, V Lundblad, E Blackburn, eds. Telomeres. 2nd Edition. Cold Spring Harbor: Cold Spring Harbor Laboratory Press. 2006:261-316.

4 Liti G, Peruffo A, James SA, Roberts IN, Louis EJ. Inferences of evolutionary relationships from a population survey of LTR-retrotransposons and telomeric-associated sequences in the Saccharomyces sensu stricto complex. Yeast 2005; 22:177192.

5 Kellis M, Patterson N, Endrizzi M, Birren B, Lander ES. Sequencing and comparison of yeast species to identify genes and regulatory elements. Nature 2003; 423:241-254.

6 Askree SH, Yehuda T, Smolikov S, et al. A genome-wide screen for Saccharomyces cerevisiae deletion mutants that affect telomere length. Proc Natl Acad Sci USA 2004; 101:86588663.

(Supplementary information is linked to the online version of the paper on the Cell Research website.) 